\title{
Strengthening Behavioral Patterns Impacting COVID-19 Vaccination and Oral Health Amidst the Looming Third Wave Scenario-Need of the Hour!
}

\author{
Deesha Kumari $^{1}$ Shilpa Mailankote ${ }^{1}$ \\ ${ }^{1}$ Department of Public Health Dentistry, Nitte (Deemed to be \\ University), AB Shetty Memorial Institute of Dental Sciences \\ (ABSMIDS), Mangalore, Karnataka, India \\ J Health Allied Sci ${ }^{\mathrm{NU}}$ 2022;12:350-355.
}

\begin{abstract}
Keywords

- COVID-19

- vaccination

- behavior

- oral health

Aim The current review aims to ascertain the scope and depth of the behavioral patterns impacting COVID-19 vaccination and oral health as well as recognize the rates of acceptance and hesitancy of coronavirus disease 2019 (COVID-19) vaccine.

Background The COVID-19 vaccination drive is a decisive factor in the control and termination of the COVID-19 pandemic. Concerns about vaccine hesitancy are growing worldwide and it has been thus declared as one among the top 10 threats in 2019 by the World Health Organization; thus, addressing the obstacles and expediters of vaccines will be fundamental in initiating and effectively implementing the interventions to accomplish extreme vaccine coverage.

Review Results The vaccine utility is not merely reliant on the efficacy and the safety of the vaccine. The major factor is the vaccine acceptance among the general public of all the sectors of the society. Trust in public authorities, adverse effects, phobias, community influences affect the vaccine acceptance rate tremendously. With an evolving third peak of the COVID pandemic, the affinity toward availing COVID vaccine has increased but the hesitancy rate among individuals still persists.

Conclusion The large variations in vaccine acceptance and hesitancy rates can impact the efforts put forth in eliminating the disease. Thus public health policies can enable development of effective approaches to overcome the hesitancy related to COVID-19 vaccine thereby increasing its uptake and facilitating smooth functioning of the vaccination drives which could help conduit the existing gaps.

Clinical Significance Involvement of dental health professionals in vaccination campaigns is vital for the enforcement of common risk factor approach wherein focus can be shed on both general and oral health wellness.
\end{abstract}

\begin{abstract}
Address for correspondence Shilpa Mailankote, MDS, Department of Public Health Dentistry, Nitte (Deemed to be University), AB Shetty Memorial Institute of Dental Sciences (ABSMIDS), Mangalore, Karnataka 575018, India (e-mail: shilpasharma17@gmail.com).
\end{abstract}

published online January 18,2022
DOI https://doi.org/ $10.1055 / \mathrm{s}-0041-1741432$ ISSN 2582-4287.

\footnotetext{
(c) 2022. Nitte (Deemed to be University). All rights reserved. This is an open access article published by Thieme under the terms of the Creative Commons Attribution-NonDerivative-NonCommercial-License, permitting copying and reproduction so long as the original work is given appropriate credit. Contents may not be used for commercial purposes, or adapted, remixed, transformed or built upon. (https://creativecommons.org/ licenses/by-nc-nd/4.0/) Thieme Medical and Scientific Publishers Pvt. Ltd., A-12, 2nd Floor, Sector 2, Noida-201301 UP, India
} 


\section{Background}

The black swan event of the novel coronavirus disease 2019 (COVID-19) has disrupted the lives of millions globally. The outbreak was first reported in a seafood wholesale wet market in Wuhan, Hubei Province, China in December 2019. The disease affected around $66 \%$ of the staff who presented with gastrointestinal symptoms. ${ }^{1}$ The disease later spread to various nations due to international travelers. The outbreak was declared as a Public health emergency by the World Health Organization on January 30, 2020. ${ }^{1}$

A per the data available worldwide, the case fatality rate of this pandemic has been observed to be $4 \%$ but may vary from 0 to $20 \%$ between countries. ${ }^{2}$ The variation in the fatality rate may be attributed to the population composition, gender variations, and presence of co-morbidities. It has been observed that males had a higher death rate than females. In addition, individuals with diabetes, lung disease, and hypertension were 3.38 to 3.73 times more prone to witness fatality than their healthy counterparts. ${ }^{3}$ As of May 2021, more than 17 crore people have been affected by the disease with over 35.4 lakh people succumbing to death in over 200 nations globally. India, which witnessed a major second wave of the disease in mid-February 2021, has reported the second highest number of cases of the disease.

Apart from rising mortality rates, the morbidity associated with the pandemic cannot be overlooked. COVID-19 has had a significant impact on the general health of individuals which affects their day to day functions. Though the disease primarily affects the lungs, the effects of the virus penetration can be manifested in various parts of the human body like heart, kidneys and brain due to vascular permeability. ${ }^{4}$ Studies showed that a significant viral load can be observed in the nasal and oral cavity. Thus, one cannot flout the effects of COVID-19 on the oral cavity. In addition, inflammatory markers like C-reaction proteins are also elevated in patients infested with the disease. These markers have been shown to be significantly associated with periodontal diseases and other oral problems. ${ }^{5}$ This multitude of factors necessitates the need for voluminous interventions to intervene the spread of the disease.

Amongst the various measures taken to combat the spread of the virus, the government of India imposed stringent lockdown measures with emphasis on personal hygiene measures like use of mouth mask, frequent hand washing with soap and water, and use of sanitizer. In addition, India being one of the biggest pharmaceutical manufacturing capacities in the world was obligated to play a prime role in the vaccination drive against COVID-19.

Thus, with the aim to contain the disease at the earliest, vaccine trials were implemented in full swing which resulted in the successful discovery of two vaccines that cleared the phase 1 and 2 trials. The vaccines were then approved for administration in population groups which led to the initiation of the vaccination drive in India. However, the acceptance rate of the vaccine among population groups was trivial owing to various reasons. This review thus aims at assessing the factors that influenced the vaccination behavior and oral health of individuals.

\section{The Tale of Indian Vaccine Production}

India's vaccination production involved indigenous vaccines and also collaborative production wherein pharmaceutical and biotech companies tied up with international vaccine developers. Thus there were eight leading vaccine candidates which were undergoing clinical trials in India (-Fig. 1). Covishield was produced by the Serum Institute of India which was primarily a version of the Oxford UniversityAstraZeneca vaccine consisting of a replication-deficient chimpanzee adenoviral vector ChAdOx1 having the SARSCoV-2 structural surface glycoprotein antigen gene. The phase 1 clinical trials of the original vaccine were initiated in United Kingdom, Brazil, South Africa, and Kenya that showed to have an efficacy rate of $70.4 \% .{ }^{6}$ With successful results at phase I and II trials, the vaccine entered the phase III trials in mid-November. In India, the II/III clinical trials were conducted in 14 centers among 1,600 participants for comparison of the safety of Covishield with the original Oxford manufactured vaccine. The obtained data were comparable to the results of the previous trials. ${ }^{7}$

On the other hand, Covaxin is India's first home manufactured vaccine against the deadly COVID-19. This vaccine was developed by Bharat Biotech in collaboration with the Indian Council of Medical Research (ICMR) and the National Institute of Virology, Pune. This vaccine contains inactivated virus, developed in Vero cells. A total of 800 participants were included in the phase I as well as phase II trials wherein a strong immunological response and protection were observed. The third phase of the vaccine trials was developed in November 2020 on 25,800 participants in 25 centers in India which was one of the largest clinical trials in India and showed to have an interim efficacy of $81 \%^{7}$

Among the few domestic vaccines which are still in the phase II trials are the ZyCoV-D and two unnamed vaccines. ZyCoV-D is developed by Ahmedabad-based Cadila Healthcare. The vaccine is based on the technology of plasmid DNA vaccination. However, the vaccine is still in the phase II trials. ${ }^{7}$ The first unnamed vaccine is developed by the Biological E. Ltd., together with Dynavax Technologies Corporation as well as Baylor College of Medicine. This is on the basis of the Classica Vaccine Technology, which uses a protein antigen, SARS-CoV-2 Spike RBD, which is adsorbed onto an adjuvant called Alhydrogel (Alum), by combining with one more accepted adjuvant, CpG 1018. This vaccine is also in phase II trials. The second unnamed vaccine is an mRNA vaccine which has been developed by Gennova Biopharmaceuticals Ltd., Pune in partnership with HDT Biotech Corporation of the United States. ${ }^{7}$

Yet another vaccine is Gam-COVID-Vac which is developed under the trade name Sputnik V. This two-vector viral vaccine based on human adenovirus Ad5 and Ad26 is developed by Gamaleya National Centre of Epidemiology and Microbiology of Moscow in Russia. ${ }^{7}$ The clinical trials of the vaccine at 25 hospitals in Russia among 21,977 


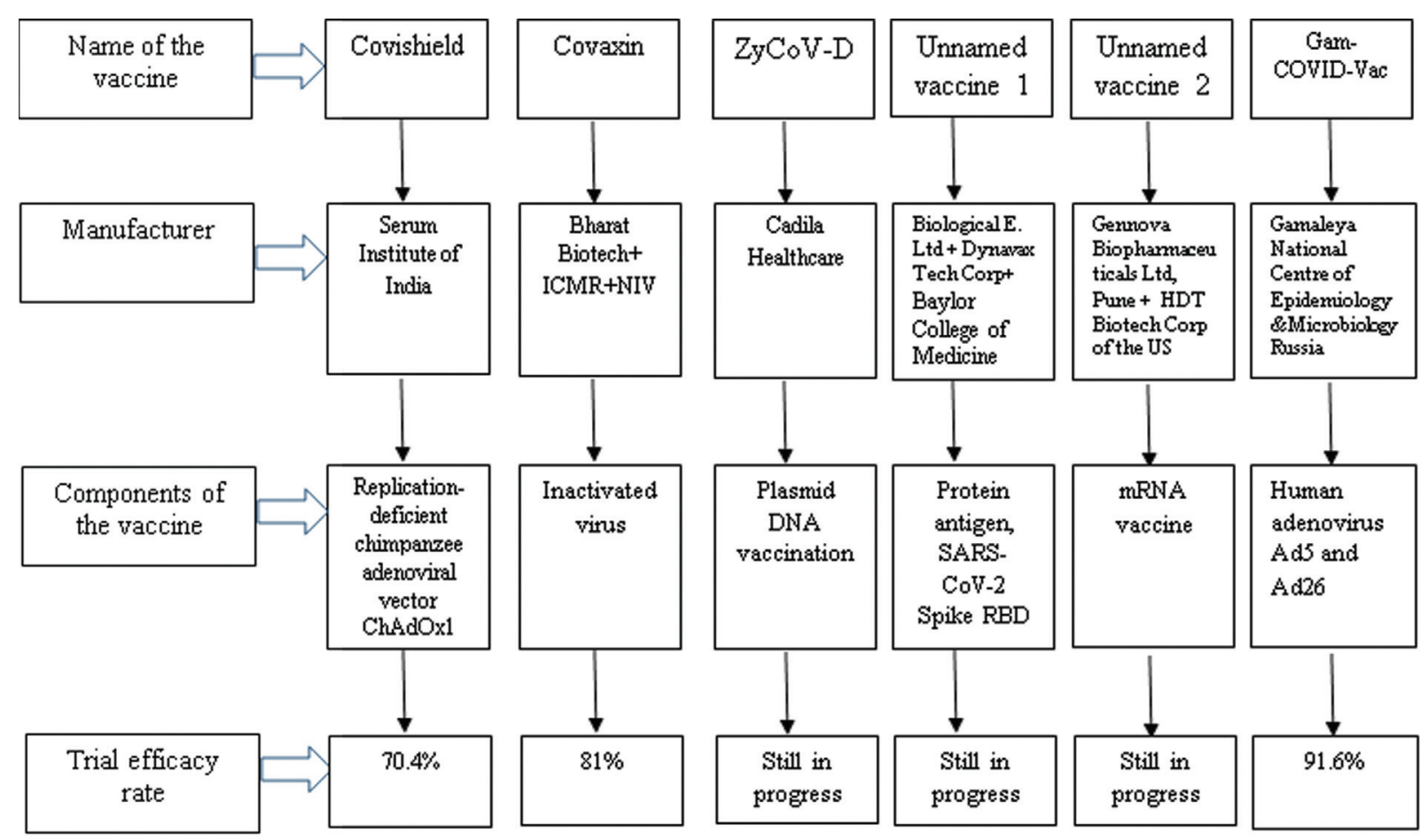

Fig. 1 Flowchart indicating the characteristics of different vaccines available in India.

participants showed to have an efficacy rate of $91.6 \% .^{8}$ The vaccine has been granted approval from the DCGI for testing and production.

\section{Rate of Acceptance of COVID-19 Vaccination}

India's largest vaccination drive against COVID-19 was initiated on January 16, 2021. Based on the strategy of Central Government, health care workers and frontline health workers were the first listed out to be vaccinated as they were at high risk of contracting the disease. Following this, people aged 60 years and above and those with co-morbidities were included in the vaccination process. From April $1^{\text {st }}$, people aged 45 years and above were further included. ${ }^{9}$ A COVID-19 Symptom Survey was conducted by the University of Maryland and Carnegie Mellon University in partnership with Facebook to assess aspects related to COVID-19. As per the reports $45 \%$ of the population was definitely ready to get vaccinated whereas $12.4 \%$ of the population was definitely not ready to get vaccinated. ${ }^{10}$ The acceptance of COVID-19 vaccination among the Indian population was found to be good. Various studies have shown that the acceptance rate varied from 41 to $81.3 \%$ among population groups. ${ }^{11,12} \mathrm{~A}$ global survey analyzing the acceptance rate of COVID-19 vaccine among 10 low and middle income countries have shown that the vaccine acceptance rate was $80.3 \% .{ }^{13}$ However, as of August 2021, only $8.9 \%$ of the total population have been vaccinated in India. $30.9 \%$ of the population have received at least one dose of the vaccine. ${ }^{9}$ According to the A COVID-19 Symptom Survey, Kerala displayed the highest number of people who were unwilling to get themselves vaccinated. ${ }^{10}$ The above data are suggestive of the fact that the attitude of people toward COVID-19 vaccination varies which impacts the rate of vaccination.

\section{Factors Affecting the Attitude of People toward COVID Vaccination}

The acceptance level of COVID-19 vaccination by people has been influenced by various factors like demographic, personal, community, and environmental characteristics. An array of multifactorial elements with varying effects on different population groups was observed through individual studies conducted.

Among the demographic factors, age, gender, occupation, and educational status can be considered as prime factors which have influenced the acceptance rate of COVID-19 vaccination among people. As of August 2021, 175 million people aged 18 to 44 years, 111 million people aged $>45$ years, and 78 million people aged $>60$ years have received at least one dose of the vaccination. ${ }^{14}$ Numerous surveys conducted have shown that age has been significantly associated with the COVID-19 vaccine acceptance or hesitance rate. It has been found that the acceptance rate of the people aged $>45$ years was lower as compared with the younger age groups. ${ }^{15,16}$ However, a global survey conducted has found that people aged 25 years and above were more likely to accept the vaccine than those aged 18 years and below. ${ }^{13,17}$ This could be attributed to the varying degrees of awareness among the people regarding the vaccine. Another demographic characteristic determining the acceptance rate of COVID-19 vaccination is gender. Though the differences were small, it was observed that females were more willing to accept the vaccine as compared with males. ${ }^{13,15,17,18}$ This 
could be due to the fact that women are more inclined toward availing preventive care and bear the responsibility of the health care for the family. In addition, the ideology of men being stronger and the conservative nature may be the driving force for this behavior, even amidst the higher rate of men succumbing to death due to COVID-19. ${ }^{18}$

The socioeconomic status of the population groups expressed through education, occupation, and family income were also seen to have an effective role in the COVID-19 vaccine acceptance behavior. Individuals belonging to higher socio-economic status were significantly associated with higher vaccine acceptance rate than individuals from the lower socio-economic status. ${ }^{10,15,17,19}$ This can be attributed to the fact that individuals with a higher level of education with a professional job status and income were more inclined to avail the COVID vaccine due to the ease and affordability of access to health care services.

On the other hand, personal or individual factors also seem to bear a prime role in vaccine acceptance rates of people. Various descriptive studies conducted across population groups have suggested that fear of health due to vaccine allergic reactions, incredulity over vaccine trails, nationality of the vaccine produce, rush in vaccine production, exaggeration of COVID-19 prevalence, and incidence data may be cited as the few reasons affecting the uptake of COVID vaccine by people. ${ }^{19}$ Studies have shown that around $44 \%$ of the population were apprehensive about the possible side effects and 29\% of the population showed concerns regarding the clinical trials of the vaccine since the vaccine for COVID-19 was delivered at a faster pace amid various speculations that enhance the fear among people regarding the vaccine. ${ }^{20}$

The efficacy rate of the Indian manufactured vaccine as compared with the foreign manufactured vaccines could also be a contributing factor. The efficacy rate of $94 \%$ of foreign vaccines as compared with the $74 \%$ efficacy of Indian vaccines could produce a lag in the minds of people to vaccinate themselves with the available vaccine or wait for the best to be available in the market. Around $67 \%$ of the participants in surveys conducted have shown that people wish to get themselves vaccinated with a vaccine which has a higher efficacy rate. ${ }^{21}$ However, around $33 \%$ of the population preferred vaccines developed by Indian pharmaceutical companies. ${ }^{18}$

Another important factor is the cost of the vaccines. At present, vaccine is available at private and government health care sectors. While the government-based health sectors are providing vaccine free of cost, the private health care sectors are charging rupees 750 to 1,300 per dose of the vaccine, based on the vaccine type. ${ }^{9}$ Thus, $20 \%$ of the population comprehend cost of the vaccine as a barrier in availing vaccine, as it is an out of pocket expense and a burden to the family disbursements. ${ }^{18}$ On the personal front, the time can also be perceived as a significant barrier in availing vaccine. COVID-19 vaccine demand and supply faced an inverse relationship wherein the demand for the vaccine upsurged the supply of the vaccine, even though the production was carried on a large phase. This eventually steered a situation wherein people are forced to wait for long hours outside the health centers to avoid missed vaccine schedules. This brunt cannot be faced by working individuals and thus can affect the vaccine acceptance behavior of individuals. ${ }^{12,18}$

The vaccine behavior, though not limited to COVID vaccine alone can largely be attributed to the individual's phobia of needles and fear of adverse effects. Needle phobia/trypanophobia affects around 10 to $25 \%$ of individuals across all age groups. ${ }^{22,23}$ The drive for COVID vaccination in the country can be hindered by needle phobics who are likely to refuse the vaccination, as it involves not one but two jabs. In addition, around $73 \%$ of people expressed their concerns regarding the risks or adverse effects associated with the COVID vaccine. ${ }^{18}$ The common effects seen after the vaccination range from mild symptoms like fever, muscle pain to severe symptoms like breathlessness. Thus, the response of peers, family, friends. and relatives who are already vaccinated could endure a significant influence on the minds of people who are unwilling to take vaccine. ${ }^{20}$

The perceived risk of COVID infection and the belief in selfimmunity can also be the other driving factors in this vaccination initiative. Around 22 to $30 \%$ of the population perceived to be at lesser risk of COVID infection. ${ }^{12,18,19}$ This could be due to the self-perceived immunity of people which renders them the thought of being resistant to COVD-19 infection. The information in social media and through peers on a large basis, initiated the belief that increasing the immunity of an individual through household remedies can help them combat the infection on a large basis rather than depending on a vaccine.

In addition, trust on public authorities was also perceived as a reason of hesitancy toward availing COVID vaccine. Surveys conducted have demonstrated that individuals who trusted the government were 1.6 times more willing to avail the vaccine as compared with the individuals who had no faith in the government. ${ }^{17,18}$ Trust plays a key role in shaping the public opinion regarding vaccines. However, the seeming reluctance among individuals may arise above science of the vaccines and may be determined by the politics driving the production and availability of the vaccine. This could be due to the known facts like political differences and statements passed by oppositions to demean the reigning party, consistent changes in vaccine schedules which could create a restraint in the minds of people regarding the vaccination process.

\section{Will COVID-19 Vaccination Ace Oral Health and Access to Oral Health Care Services?}

The impact of COVID-19 on oral health can be viewed in a bidirectional manner. On one hand, COVID-19 can affect the oral tissues and instigate several oral diseases and on the other hand, the rising cases and evolving strains of the virus can impede the oral health care availing behavior of individual, due to the fear of contracting the disease. As per numerous research, the neurotropic and mucotropic properties of the SARS-CoV-2 virus can significantly affect the salivary gland functions, alter the taste sensations, integrity 
of the oral mucosa causing an imbalance in the oral environment dynamics. ${ }^{24}$ Individuals can thus be predisposed to a variety of oral problems like xerostomia, opportunistic fungal infections, ulcerations, and gingival infections. ${ }^{5,24}$ The cytokine storm that is caused by the impaired humoral mechanism and cellular mechanism can magnify the autoimmune disorders well within the oropharyngeal region, which can be of great concern. ${ }^{24}$ Apart from the virus impingement, the after effects of the treatment can also predispose the individuals to various infections. Uncontrolled diabetes mellitus or systemic illness and rampant use of corticosteroids during the treatment of COVID-19, extensive use of ventilation and oxygen therapies in the background appear to be silent triggers in the rising cases of mucormycosis. ${ }^{24}$ India appears to account for around $81 \%$ of the total cases of mucormycosis, wherein $88.9 \%$ of the cases are seen involving the nose and sinus area and $56.7 \%$ cases involve the rhino-orbital region. ${ }^{25}$

However, with the increasing rates of COVID-19 cases, the decrease in patients availing dental care is also alarming. Studies have reported that around $97 \%$ of dentists have reported the decline in patient attendance for availing oral care procedures. An inverse trend was noted between the COVID-19 pandemic prevalence and oral health care admissions, which further increased during the second wave of the pandemic. $^{26,27}$ Research suggests that in view of the pandemic, $32.5 \%$ of the patients seeking dental care for routine dental treatments preferred teleconsultation in the present situation. However, only $18 \%$ of individuals were ready to avail dental treatment owing to the pandemic distress, since oral treatments involve aerosol procedures that can increase their risk of contracting the disease. ${ }^{28,29}$

Thus the vaccination drive can accentuate the current oral disease distress to a large extent. This can be owed to the fact that vaccinated individuals are less prone to face the serious illness or effects of the disease and thereby avoid hospitalization and death. Reports suggest that the attack rate of the disease sharply decreased from 9 to $4 \%$ post vaccination. ${ }^{30}$ In addition, the immunity boost acquired by the vaccination process, be it natural or psychological, can enhance the individual's impetus to attain oral health care on a regular basis.

\section{Recommendations}

Amidst the panic of the third wave of the COVID-19 pandemic, the COVID vaccine is definitely a ray of silver lining for a prospective future. The vaccination drive in India is a multifactorial enterprise which is largely dependent on the attitude of individuals to the vaccine. Individuals with different ideological objections, the accessibility, affordability and political factors largely affect the acceptance rate of people. The safety concerns and the costs associated with the vaccine are highly associated with the refusal tendencies of individuals. Thus, it is the need of the hour to enhance the grass root strategies to influence all sections of the society. New advances in technology utilized for vaccine production should be highlighted, to boost the vaccine acceptance among people. Government officials and advocacy assemblies must address the vaccine hesitancy by building vaccine literacy to fasten the vaccine acceptance rates. Utilizing the health professionals who are considered as sources of trustworthy information by people, interventions like awareness campaigns via social media and other multimedia campaigns should be designed with the objective to spread transparent and trustworthy information about the safety, efficacy, and effects of the vaccine. Strategies should also involve addressing the affordability and accessibility aspects of the vaccine, by making the free vaccines available at a larger scale. Enhancement of the vaccine booking strategies, house-tohouse vaccination campaigns majorly addressing the vulnerable and geriatric population groups will also go a long way in this regard.

\section{Conclusion}

An approach based on increasing internal motivation and imbibing attitudinal changes in an individual can pave the way for a healthy vaccinated nation in our fight against this deadly COVID-19 pandemic. Dentists can play a crucial role by thus being a part of the multidisciplinary team approach and bridge the gap by reducing oral health disparities, identifying risk factors and early lesions, creating awareness and promoting COVID-appropriate behavior especially among the immunocompromised, elderly, and vulnerable populations. Utilization of dental health professionals in vaccination campaigns is vital for the enforcement of common risk factor approach wherein focus can be shed on both general and oral health wellness. Scaling up awareness campaigns and use of social media in this regard will play a major role in the success of vaccination campaigns.

\section{Authors' Contribution}

D.K. contributed toward Conceptualization of the topic, literature search, manuscript writing, and finalization. S. M. did the literature search, manuscript writing, and finalization.

\section{Funding}

None.

Conflict of Interest

None declared.

\section{References}

1 Wu Y-C, Chen C-S, Chan Y-J. The outbreak of COVID-19: an overview. J Chin Med Assoc 2020;83(03):217-220

2 Sorci G, Faivre B, Morand S. Explaining among-country variation in COVID-19 case fatality rate. Sci Rep 2020;10(01):18909

3 Lu L, Zhong W, Bian Z, et al. A comparison of mortality-related risk factors of COVID-19, SARS, and MERS: a systematic review and meta-analysis. J Infect 2020;81(04):e18-e25

4 Guven G, Ince C, Topeli A, Caliskan K. Cardio-pulmonary-renal consequences of severe COVID-19. Cardiorenal Med 2021;11(03): 133-139

5 Kamel AHM, Basuoni A, Salem ZA, AbuBakr N. The impact of oral health status on COVID-19 severity, recovery period and C- 
reactive protein values. Br Dent $J 2021$ (e-pub ahead of print). Doi: 10.1038/s41415-021-2656-1

6 Voysey M, Clemens SAC, Madhi SA, et al; Oxford COVID Vaccine Trial Group. Safety and efficacy of the ChAdOx1 nCoV-19 vaccine (AZD1222) against SARS-CoV-2: an interim analysis of four randomised controlled trials in Brazil, South Africa, and the UK. Lancet 2021;397(10269):99-111

7 Kumar VM, Pandi-Perumal SR, Trakht I, Thyagarajan SP. Strategy for COVID-19 vaccination in India: the country with the second highest population and number of cases. NPJ Vaccines 2021;6 (01):60

8 Logunov DY, Dolzhikova IV, Shcheblyakov DV, et al; Gam-COVIDVac Vaccine Trial Group. Safety and efficacy of an rAd26 and rAd5 vector-based heterologous prime-boost COVID-19 vaccine: an interim analysis of a randomised controlled phase 3 trial in Russia. Lancet 2021;397(10275):671-681

9 MoHFW Ministry of Health and Family Welfare. Vaccination State Data. Accessed August 16, 2021 at: https://www.mohfw.gov.in/

10 Chowdhury S, Motheram A. Covid-19 vaccine hesitancy: trends across states, over time. Ideas for India. Accessed August 16, 2021 at: http://www.ideasforindia.in/topics/governance/covid-19vaccine-hesitancy-trends-across-states-over-time.html

11 Bhargava R, Jain G, Bhargava A, Gupta S. COVID-19 vaccination drive: impact on the acceptance of vaccine among the general population of India. J Manage Res Anal 2021;8(02):61-69

12 Das B, Padhye A. Public perception and potential acceptance of COVID-19 vaccine in India. Public health review. Int J Public Health Res 2021;8(02):23-31

13 Solís Arce JS, Warren SS, Meriggi NF, et al. COVID-19 vaccine acceptance and hesitancy in low- and middle-income countries. Nat Med 2021;27(08):1385-1394

14 MoHFW. COVID-19 Vaccination Update. Accessed August 29, 2021 at: https://pib.gov.in/pib.gov.in/Pressreleaseshare.aspx?PRID=1743685

15 Islam F, Agarwalla R, Panda M, et al. Assessment of the knowledge, preferences and concern regarding the prospective COVID-19 vaccine among adults residing in New Delhi, India-a crosssectional study. J Family Med Prim Care 2021;10(06):2369-2375

16 Syed Alwi SAR, Rafidah E, Zurraini A, Juslina O, Brohi IB, Lukas S. A survey on COVID-19 vaccine acceptance and concern among Malaysians. BMC Public Health 2021;21(01):1129

17 Lazarus JV, Ratzan SC, Palayew A, et al. A global survey of potential acceptance of a COVID-19 vaccine. Nat Med 2021;27(02):225-228
18 Jacob J, Stephen S, Issac A, et al. Determinants of willingness for COVID-19 vaccine: implications for enhancing the proportion of vaccination among Indians. Cureus 2021;13(05):e15271

19 Praveen SV, Ittamalla R, Deepak G. Analyzing the attitude of Indian citizens towards COVID-19 vaccine-a text analytics study. Diabetes Metab Syndr 2021;15(02):595-599

20 Suresh A, Konwarh R, Singh AP, Tiwari AK. Public awareness and acceptance of COVID-19 vaccine: an online cross-sectional survey, conducted in the first phase of vaccination drive in India. 2021 Accessed August 31, 2021 at: https://www.researchsquare. com/article/rs-324238/v1

21 Harapan H, Wagner AL, Yufika A, et al. Acceptance of a COVID-19 vaccine in Southeast Asia: a cross-sectional study in Indonesia. Front Public Health 2020;8:381

22 Hamilton JG. Needle phobia: a neglected diagnosis. J Fam Pract 1995;41(02):169-175

23 Memon B, Hafeez-ur-Rehman, Muhammad S, Ali A. Prevalence of needle phobia among young patients presenting to tertiary care government hospitals of Karachi, Pakistan. Int J Res 2015;2:127-135

24 Dziedzic A, Wojtyczka R. The impact of coronavirus infectious disease 19 (COVID-19) on oral health. Oral Dis 2021 Apr;27 (Suppl 3):703-706

25 Singh AK, Singh R, Joshi SR, Misra A. Mucormycosis in COVID-19: a systematic review of cases reported worldwide and in India. Diabetes Metab Syndr 2021;15(04):102146

26 Ahmadi H, Ebrahimi A, Ghorbani F. The impact of COVID-19 pandemic on dental practice in Iran: a questionnaire-based report. BMC Oral Health 2020;20(01):354

27 Cagetti MG, Balian A, Camoni N, Campus G. Influence of the COVID-19 pandemic on dental emergency admissions in an urgent dental care service in North Italy. Int J Environ Res Public Health $2021 ; 18(04): 1812$

28 Farsi D, Farsi N. Mothers' knowledge, attitudes, and fears about dental visits during the COVID-19 pandemic: a cross-sectional study. J Int Soc Prev Community Dent 2021;11(01):83-91

29 Meisha DE, Alsolami AM, Alharbi GM. Social determinants of seeking emergency and routine dental care in Saudi Arabia during the COVID-19 pandemic. BMC Oral Health 2021;21 (01):212

30 Moghadas SM, Vilches TN, Zhang K, et al. The impact of vaccination on COVID-19 outbreaks in the United States. medRxiv 2021 Jan 2;2020.11.27.20240051 